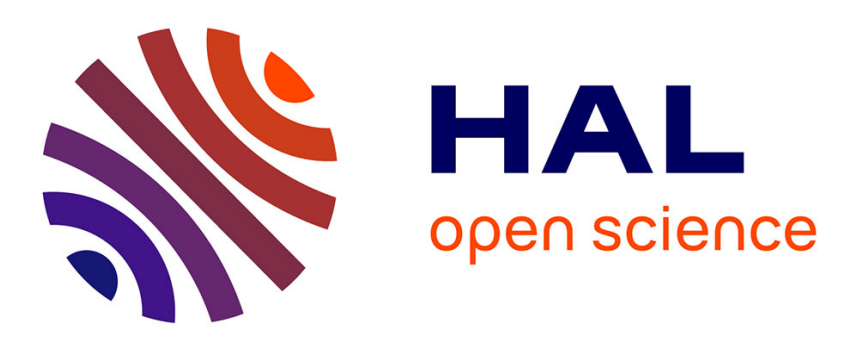

\title{
Global observers with finite-time and fixed-time convergence for a class of uncertain mechanical systems
}

\author{
Amina Benarab, W. A. Apaza-Perez
}

\section{To cite this version:}

Amina Benarab, W. A. Apaza-Perez. Global observers with finite-time and fixed-time convergence for a class of uncertain mechanical systems. 60th IEEE Conference on Decision and Control (CDC), Dec 2021, Austin, United States. hal-03323478v3

\section{HAL Id: hal-03323478 \\ https://hal.science/hal-03323478v3}

Submitted on 1 Oct 2021

HAL is a multi-disciplinary open access archive for the deposit and dissemination of scientific research documents, whether they are published or not. The documents may come from teaching and research institutions in France or abroad, or from public or private research centers.
L'archive ouverte pluridisciplinaire HAL, est destinée au dépôt et à la diffusion de documents scientifiques de niveau recherche, publiés ou non, émanant des établissements d'enseignement et de recherche français ou étrangers, des laboratoires publics ou privés. 


\title{
Global observers with finite-time and fixed-time convergence for a class of uncertain mechanical systems
}

\author{
Amina BENARAB and W. Alejandro APAZA-PEREZ
}

\begin{abstract}
This paper proposes global sliding mode observers for a class of one-degree-of-freedom mechanical systems. For the observer design, besides the usual Coriolis and centrifugal forces, we consider uncertain dry frictions and disturbances. Moreover, the system is not required to be bounded-input bounded-state stable, rendering the observer design problem challenging. The observer design exploits the specific relationships between the inertia and Coriolis terms providing a sliding-mode observer, with global theoretically exact finite-time and fixed-time convergence to the velocities of the mechanical system. The efficiency of the proposed observer is validated through simulations on an inverted pendulum.
\end{abstract}

\section{INTRODUCTION}

Control of mechanical systems usually requires information about position and velocity. Often only the position is available for which an estimation of the velocity is required, this has given rise to interesting challenges in the area of control and observation [1], [2]. The main challenge in building observers to estimate the velocity in mechanical systems is the presence of nonlinear terms (e.g. Coriolis and centrifugal forces) and disturbances (e.g. unmodeled dynamics). The sliding-mode observers/differentiators [3], [4], [5], [6], [7] provide theoretically exact finite-time and fixed-time convergence despite the presence of bounded disturbances when the system has bounded-input boundedstate (BIBS) stability, therefore they are semi-global. If the BIBS property is not satisfied, then finite-time/fixed-time convergence is not guaranteed, see an illustrative example in [8].

In this paper, we will consider mechanical systems of one degree of freedom (1-DOF) with disturbances, which could not have the BIBS property.

State of art and motivation:

1) If the dynamical systems with disturbances satisfying the conditions for the existence of an observer, among them the relative-degree-one condition [9], [10], then dissipative observers [11], [12], which include high-gain/Lipschitz observers, result to be efficient to deal with BIBS restriction. They are able to estimate globally and exponentially the real states using dissipative properties which could have the nonlinear terms. But mechanical systems with disturbances have the relative degree greater than one, with respect to

A. Benarab is with the Université Paris-Saclay, CNRS, CentraleSupélec, Inria, Laboratoire des signaux et systèmes, 91190, Gif-sur-Yvette, France, with Institut Polytechnique des Sciences Avancées, 94200 Ivry-sur-Seine, France. amina.benarabecentralesupelec.fr

W.A. Apaza-Perez is with the Université Paris-Saclay, CNRS, CentraleSupélec, Laboratoire des signaux et systèmes, 91190, Gif-sur-Yvette, France willy-alejandro. apaza-perezel2s. centralesupel ec.fr (w.r.t.) the measured position.

2) There are many works (e.g. see [13], [14], [15], [16]) dealing with the Coriolis and centrifugal forces, which provide global observers when the model of the mechanical system is completely known. Through state transformations, [15], [16] propose observers with fairly high dimension, namely $3 n+1$ and $2 n+2$ respectively, where $n$ is the dimension of the unmeasured velocity. [13], [14] propose observers with the same dimension of the system for a class of mechanical systems. However, the challenge of dealing with frictions, disturbances, and obtaining an estimation theoretically exact of velocity was not considered.

3) To overcome the BIBS restriction and the presence of Coriolis and centrifugal forces some recent works have been presented. [8] and [17] propose a finite-time sliding-mode observer for 1-DOF mechanical systems with disturbances and uncertain inertial matrix, respectively, using state transformations inspired by [13]. An extension to the 2-DOF was proposed in [18] where some assumptions in the inertia matrix are required. But, global observers with convergence in fixed time have not been considered by previous works.

Main contribution. A global sliding mode observer for a class of mechanical systems with Coriolis and centrifugal forces, dry frictions, and bounded disturbances is proposed. The proposed observer does not require a prior state transformation and provides finite-time and fixed-time convergence to the position and velocity of the mechanical system. BIBS stability of the mechanical system is not required, and its gains can be obtained from a feasible matrix inequality.

Some definitions and preliminary results are described in Section II. The problem statement is presented in Section III. In Section IV, the observer design for the finite-time/fixedtime convergences is described, with the main results. Finally, to illustrate our results, numerical simulations for an inverted pendulum on a cart are shown in Section V.

\section{PReliminaries}

Notations. Symbols $\mathbb{R}, \mathbb{R}_{\geq 0}, \mathbb{R}_{>0}$ denote the set of real, of nonnegative real numbers, of positive real numbers, respectively. $|\cdot|$ denotes the absolute value in $\mathbb{R}$ and $\|\cdot\|$ denotes the Euclidean norm on $\mathbb{R}^{n}$. Let $\lambda_{\min }(P)$ and $\lambda_{\max }(P)$ be respectively the minimum and the maximum eigenvalues of the positive definite symmetric matrix $P \in \mathbb{R}^{n \times n}$ and $P^{T}$ defines the transposed matrix of $P$.

Define the signed power function $\lceil x\rfloor^{r}$, for any $x \in \mathbb{R}$ and $r \in \mathbb{R}_{\geq 0}$, as an increasing odd function defined as $\lceil x\rfloor^{r}=$ $|x|^{r} \operatorname{sign}(x)$, where $\operatorname{sign}(x)$ is the standard sign function. The function $\lceil x\rfloor^{r}$ satisfies following properties: 
- For each $r \in[0,1),\lceil x\rfloor^{r}$ is differentiable for all $x \neq 0$, $\lceil x\rfloor^{r} \in C^{1}$ if $r \in[1,2]$ and $\lceil x\rfloor^{r} \in C^{2}$ if $r \in(2, \infty)$.

- $\frac{\mathrm{d}}{\mathrm{d} x}\lceil x\rfloor^{r}=r|x|^{r-1}$ and $\frac{\mathrm{d}}{\mathrm{d} x}|x|^{r}=r\lceil x\rfloor^{r-1}$, for all $x \neq 0$ and $r \neq 0$.

- For any $r, s \in \mathbb{R}_{\geq 0},\lceil x\rfloor^{r}\lceil x\rfloor^{s}=|x|^{r+s}$ and $|x|^{r}\lceil x\rfloor^{s}=$ $\lceil x\rfloor^{r+s}$ and $\lceil x\rfloor^{0}=\operatorname{sign}(x)$.

\section{A. Finite-time and fixed-time stability}

Consider the following system

$$
\dot{x}(t)=f(t, x(t)), \quad t>t_{0} \quad x\left(t_{0}\right)=x_{0},
$$

where $x \in \mathbb{R}^{n}$ is the state vector and $f: \mathbb{R}_{+} \times \mathbb{R}^{n} \rightarrow \mathbb{R}^{n}$ is a nonlinear continuous vector field. Assume that the origin is an equilibrium point of (1), i.e. $f(0)=0$.

Definition 1 ([19]): The origin $x=0$ of system (1) is said to be globally Finite-Time Stable if it is globally asymptotically stable and any solution $x\left(t, x_{0}\right)$ of $(1)$ reaches the equilibria at some finite-time moment, i.e. $x\left(t, x_{0}\right)=0$ $\forall t \geq T\left(x_{0}\right)$ where $T: \mathbb{R}^{n} \rightarrow \mathbb{R}_{\geq 0}$ is the so-called the settlingtime function.

Definition 2 ([20]): The origin $x=0$ of system (1) is said to be globally Fixed-Time Stable if it is globally finite-time stable and the settling time function $T$ is bounded by some positive number $T_{\max }>0$, i.e. $T\left(x_{0}\right) \leq T_{\max }$ for $\forall x_{0} \in \mathbb{R}^{n}$.

We recall some technical results and well known properties of continuous homogeneous functions.

Lemma 3 (Young's inequality [21]): For any positive real numbers $a>0, b>0, c>0, p>1$ and $q>1$, with $\frac{1}{p}+\frac{1}{q}=1$, the following inequality holds

$$
a b \leq \frac{c^{p}}{p} a^{p}+\frac{c^{-q}}{q} b^{q}
$$

and equality holds if and only if $a^{p}=b^{q}$.

Lemma 4 (Jensen's inequality [21]): For any real numbers $a_{1}, \ldots, a_{n}$, if $0<p<q$

$$
\left(\sum_{i=1}^{n}\left|a_{i}\right|^{p}\right)^{\frac{1}{p}} \leq\left(\sum_{i=1}^{n}\left|a_{i}\right|^{q}\right)^{\frac{1}{q}}
$$

For convenience notations, in the following we will consider $x, q, e, u, \delta$ instead of $x(t), q(t), e(t), u(t), \delta(t)$, respectively.

\section{PROBLEM STATEMENT}

Consider the following 1-DOF mechanical systems

$$
m(q) \ddot{q}+c(q) \dot{q}^{2}+\ell \cdot \operatorname{sign}(\dot{q})+g(q)=u+\tilde{\delta}
$$

where $q \in \mathbb{R}$ denotes the (measured) generalized position, $\dot{q}$ is the generalized velocity, $m(q)$ denotes the inertia term, $c(q) \dot{q}^{2}$ is Coriolis and centrifugal force, $\ell \operatorname{sign}(\dot{q})$ is a dry friction, $u$ is the measured torque, $g(q)$ denotes gravitational forces and $\tilde{\delta}$ represents the disturbance in the system.

The objective is to design an observer for the system (2) with theoretically exact global finite-time/fixed-time convergence to the real values of position and velocity.

Consider the system (2), which additionally satisfies the following assumptions:
(A-1) There exist constants $\varsigma_{m}>0$ and $\varsigma_{M}>0$ such that

$$
0<\varsigma_{m} \leq m(q) \leq \varsigma_{M}, \text { for all } q,
$$

is satisfied.

(A-2) The inertia and Coriolis functions satisfy

$$
\dot{m}(q)=2 c(q) \dot{q}
$$

(A-3) The disturbance $\tilde{\delta}(t)$ is bounded, i.e. there exists a constant $L_{\tilde{\delta}}>0$ such that $\|\tilde{\delta}(t)\| \leq L_{\tilde{\delta}}$ is satisfied.

Assumptions (A-1) and (A-2) are assumed in most of the mechanical systems [1]. Assumption (A-3) is a standard condition for the construction of the observer due to the fact that the system has the relative degree greater than one [9]

\section{OBSERVER DESIGN AND RESULTS}

Considering $q_{1}=q, q_{2}=\dot{q}$, the state space representation of (2) is given by

$$
\left\{\begin{array}{l}
\dot{q}_{1}=q_{2} \\
\dot{q}_{2}=\frac{1}{m\left(q_{1}\right)}\left(u-g\left(q_{1}\right)-c\left(q_{1}\right) q_{2}^{2}+\delta\right) \\
y=q_{1}
\end{array}\right.
$$

where $\delta=\tilde{\delta}-\ell \cdot \operatorname{sign}(\dot{q})$.

We propose the following observer ${ }^{1}$ for system (3):

$$
\left\{\begin{array}{r}
\dot{\hat{q}}_{1}=\frac{1}{\sqrt{m\left(q_{1}\right)}}\left(\sqrt{m\left(\hat{q}_{1}\right)} \hat{q}_{2}+k_{1} \psi_{1}\left(q_{1}-\hat{q}_{1}\right)\right) \\
\dot{\hat{q}}_{2}=\frac{1}{\sqrt{m\left(\hat{q}_{1}\right) m\left(q_{1}\right)}}\left(u-g\left(q_{1}\right)+k_{2} \psi_{2}\left(q_{1}-\hat{q}_{1}\right)\right) \\
-\frac{1}{m\left(\hat{q}_{1}\right)} c\left(\hat{q}_{1}\right) \hat{q}_{2}^{2}
\end{array}\right.
$$

where the correction functions $\psi_{1}$ and $\psi_{2}$ are defined by

$$
\begin{aligned}
\psi_{1}(\cdot)= & \alpha\lceil\cdot\rfloor^{\frac{1}{2}}+\beta\lceil\cdot\rfloor+\gamma\lceil\cdot\rfloor^{\frac{3}{2}}, \\
\psi_{2}(\cdot)= & \frac{\alpha^{2}}{2}\lceil\cdot\rfloor^{0}+\frac{3 \alpha \beta}{2}\lceil\cdot\rfloor^{\frac{1}{2}}+\beta^{2}\lceil\cdot\rfloor+ \\
& 2 \alpha \gamma\lceil\cdot\rfloor+\frac{5}{2} \beta \gamma\lceil\cdot\rfloor^{\frac{3}{2}}+\frac{3}{2} \gamma^{2}\lceil\cdot\rfloor^{2},
\end{aligned}
$$

with $\alpha, \beta, \gamma \geq 0$. Note that when $\gamma=0$ the structure of the generalized super-twisting algorithm [23] is recovered, and when $\beta=0$ and $\gamma=0$ the standard super-twisting algorithm [3]. The higher-order terms corresponding to $\gamma$ are introduced to improve the convergence properties of the observer and to obtain the uniformity of the convergence of the observer w.r.t. the initial conditions.

\section{A. Finite-time observer}

In this section, consider the observer (4), and the nonlinear functions $\psi_{1}$ and $\psi_{2}$ given in (5) and (6), respectively, with $\alpha>0$ and $\beta, \gamma \geq 0$.

Lemma 5: Let $C=\left[\begin{array}{ll}1 & 0\end{array}\right], B=\left[\begin{array}{ll}0 & 1\end{array}\right]^{T}$ and $L_{\delta}>0$. Then, there exist $\rho>0, \alpha>0$, a constant matrix $A=\left[\begin{array}{ll}-k_{1} & 1 \\ -k_{2} & 0\end{array}\right]$ and a symmetric and positive definite matrix $P \in \mathbb{R}^{2 \times 2}$, such that the following nonlinear matrix inequality holds.

$$
\left[\begin{array}{cc}
A^{T} P+P A+C^{T} L_{\delta}^{2} C+\rho P & P B \\
B^{T} P & -\frac{\alpha^{4}}{4}
\end{array}\right]<0 .
$$

\footnotetext{
${ }^{1}$ The solutions of the system (3) are defined in Filippov's sense (see [22])
} 
Proof: For the symmetric matrix $C^{T} L_{\delta}^{2} C+I\left(I=I_{2 \times 2}\right)$, there is a positive definite symmetric solution $P=P^{T}>0$ for the next inequality (quadratic Lyapunov inequality)

$$
A^{T} P+P A+C^{T} L_{\delta}^{2} C+I<0 .
$$

In other hand, one has

$$
0<\lambda_{\text {min }}^{2}(P) I \leq P \leq \lambda_{\max }^{2}(P) I
$$

which implies that

$$
I \geq \frac{P}{\lambda_{\max }^{2}(P)} .
$$

Substituting inequality (10) in (8), one gets

$$
P A+A^{T} P+C^{T} L_{\delta}^{2} C+\rho P<0
$$

where $\rho=\frac{1}{\lambda_{\max }^{2}(P)}$. For $\alpha>0$ sufficiently large the following inequality is satisfied

$$
-\frac{\alpha^{4}}{4}+B^{T} P\left[P A+A^{T} P+C^{T} L_{\delta}^{2} C+\rho P\right]^{-1} P B<0 .
$$

Finally, by Schur complement one can deduce that (7) holds.

Theorem 6: If the parameters $\beta, \gamma$ are not negative and $k_{1}$, $k_{2}, \alpha$ are selected such that (7) is satisfied, then the system (4) is a global finite-time observer for the system (3) in the sense that the estimation states $\hat{q}_{1}$ and $\hat{q}_{2}$ converge exactly in finite time to the states $q_{1}$ and $q_{2}$ of system (3).

Proof: Define the error estimation of the systems (3) and (4) as:

$$
\begin{aligned}
& e_{1}=q_{1}-\hat{q}_{1} \\
& e_{2}=\sqrt{m\left(q_{1}\right)} q_{2}-\sqrt{m\left(\hat{q}_{1}\right)} \hat{q}_{2}
\end{aligned}
$$

and its dynamic error is given by

$$
\left\{\begin{array}{l}
\dot{e}_{1}=\frac{e_{2}-k_{1} \psi_{1}\left(e_{1}\right)}{\sqrt{m\left(q_{1}\right)}} \\
\dot{e}_{2}=\frac{1}{\sqrt{m\left(q_{1}\right)}}\left(-k_{2} \psi_{2}\left(e_{1}\right)+\delta\right)
\end{array}\right.
$$

where $\dot{m}\left(q_{1}\right)=2 c\left(q_{1}\right) q_{2}$ and $\dot{m}\left(\hat{q}_{1}\right)=2 c\left(\hat{q}_{1}\right) \hat{q}_{2}$ are satisfied by Assumption (A-2).

Consider a homeomorphism $\eta$ (from $\left[e_{1} e_{2}\right]$ ) defined as $\eta=\left[\psi_{1}\left(e_{1}\right) e_{2}\right]^{T}$ and a quadratic Lyapunov function

$$
V_{1}(\eta)=\eta^{T} P \eta
$$

where $P=P^{T}>0$ is obtained from the matrix inequality (7). The time derivative of $\eta$ with $e_{1} \neq 0$ is given by

$$
\begin{aligned}
\dot{\eta} & =\left[\begin{array}{ll}
\psi_{1}^{\prime}\left(e_{1}\right) \dot{e}_{1} & \dot{e}_{2}
\end{array}\right]^{T} \\
& =\frac{\psi_{1}^{\prime}\left(e_{1}\right)}{\sqrt{m\left(q_{1}\right)}}\left(A \eta+B \frac{\delta}{\psi_{1}^{\prime}\left(e_{1}\right)}\right)
\end{aligned}
$$

where $\psi_{1}^{\prime}\left(e_{1}\right)$ denotes the derivative with respect to $e_{1}$, $\psi_{2}\left(e_{1}\right)=\psi_{1}^{\prime}\left(e_{1}\right) \psi_{1}\left(e_{1}\right), A=\left[\begin{array}{cc}-k_{1} & 1 \\ -k_{2} & 0\end{array}\right]$ and $B=\left[\begin{array}{l}0 \\ 1\end{array}\right]$.
The time derivative of $V_{1}$ along trajectories (11):

$$
\begin{aligned}
\dot{V}_{1}(\eta) & =\dot{\eta}^{T} P \eta+\eta^{T} P \dot{\eta} \\
& =\frac{\psi_{1}^{\prime}\left(e_{1}\right)}{\sqrt{m\left(q_{1}\right)}}\left(\eta^{T}\left(A^{T} P+P A\right) \eta+\eta^{T} P B \frac{\delta}{\psi_{1}^{\prime}\left(e_{1}\right)}\right. \\
& \left.+\frac{\delta}{\psi_{1}^{\prime}\left(e_{1}\right)} B^{T} P \eta\right) \\
& =\frac{\psi_{1}^{\prime}\left(e_{1}\right)}{\sqrt{m\left(q_{1}\right)}}\left[\begin{array}{c}
\eta \\
\frac{\delta}{\psi_{1}^{\prime}\left(e_{1}\right)}
\end{array}\right]^{T}\left[\begin{array}{cc}
A^{T} P+P A & P B \\
B^{T} P & 0
\end{array}\right]\left[\begin{array}{c}
\eta \\
\frac{\delta}{\psi_{1}^{\prime}\left(e_{1}\right)}
\end{array}\right]
\end{aligned}
$$

Note that nonlinear correction term $\psi_{2}$ defined in (6) satisfies

$$
\begin{aligned}
\left|\psi_{2}\left(e_{1}\right)\right|=\mid & \left.\mid e_{1}\right\rfloor^{0}\left(\frac{\alpha^{2}}{2}+\frac{3 \alpha \beta}{2}\left|e_{1}\right|^{\frac{1}{2}}+\beta^{2}\left|e_{1}\right|+\frac{3}{2} \gamma^{2}\left|e_{1}\right|^{2}\right. \\
& \left.+2 \alpha \gamma\left|e_{1}\right|+\frac{5}{2} \beta \gamma\left|e_{1}\right|^{\frac{3}{2}}\right) \mid \\
\geq & \frac{\alpha^{2}}{2}
\end{aligned}
$$

which implies

$$
\left(\frac{\delta}{\psi_{1}^{\prime}\left(e_{1}\right)}\right)^{2}=\left(\frac{\delta}{\psi_{2}\left(e_{1}\right)} \psi_{1}\left(e_{1}\right)\right)^{2} \leq\left(\frac{2 L_{\delta}}{\alpha^{2}}\right)^{2} \psi_{1}^{2}\left(e_{1}\right)
$$

with $|\delta| \leq L_{\delta}$. The inequality (14) can be written as a matrix inequality in terms of $\eta$ and $\frac{\delta}{\psi_{1}^{\prime}\left(e_{1}\right)}$

$$
0 \leq\left[\begin{array}{c}
\eta \\
\frac{\delta}{\psi_{1}^{\prime}\left(e_{1}\right)}
\end{array}\right]^{T}\left[\begin{array}{cc}
C^{T} L_{\delta}^{2} C & 0 \\
0 & -\left(\frac{\alpha^{2}}{2}\right)^{2}
\end{array}\right]\left[\begin{array}{c}
\eta \\
\frac{\delta}{\psi_{1}^{\prime}\left(e_{1}\right)}
\end{array}\right] .
$$

Adding the inequality (15) in the time derivative of Lyapunov function $V_{1}(\eta)$, we obtain

$$
\begin{aligned}
\dot{V}_{1}(\eta) & \leq \frac{\psi_{1}^{\prime}\left(e_{1}\right)}{\sqrt{m\left(q_{1}\right)}}\left[\begin{array}{c}
\eta \\
\frac{\delta}{\psi_{1}^{\prime}\left(e_{1}\right)}
\end{array}\right]^{T}\left[\begin{array}{cc}
A^{T} P+P A+C^{T} L_{\delta}^{2} C & P B \\
B^{T} P & -\left(\frac{\alpha^{2}}{2}\right)^{2}
\end{array}\right]\left[\begin{array}{c}
\eta \\
\frac{\delta}{\psi_{1}^{\prime}\left(e_{1}\right)}
\end{array}\right] \\
& \leq-\rho \frac{\psi_{1}^{\prime}\left(e_{1}\right)}{\sqrt{m\left(q_{1}\right)}} V_{1}(\eta) \quad(\text { from }(7)) \\
& \leq-\frac{\rho}{\sqrt{m\left(q_{1}\right)}}\left(\frac{\alpha}{2}\left|e_{1}\right|^{-\frac{1}{2}}+\beta\right) V_{1}(\eta) .
\end{aligned}
$$

Note that

$$
\begin{gathered}
\alpha\left|e_{1}\right|^{\frac{1}{2}} \leq\left|\left\lceil e_{1}\right\rfloor^{0}\left(\alpha\left|e_{1}\right|^{\frac{1}{2}}+\beta\left|e_{1}\right|+\gamma\left|e_{1}\right|^{\frac{3}{2}}\right)\right| \\
\leq\left|\psi_{1}\left(e_{1}\right)\right| \leq\|\eta\| \leq \frac{V_{1}^{\frac{1}{2}}(\eta)}{\lambda_{\min }(P)}
\end{gathered}
$$

holds, which is given from (9) and implies in (16) the next inequality holds

$$
\dot{V}_{1}(\eta) \leq-\frac{\rho \alpha^{2} \lambda_{\min }(P)}{2 \sqrt{m\left(q_{1}\right)}} V_{1}^{\frac{1}{2}}(\eta)-\frac{\rho \beta}{\sqrt{m\left(q_{1}\right)}} V_{1}(\eta)
$$

Note that trajectories of the estimation error dynamics cannot stay on the set $S=\left\{\left(e_{1}, e_{2}\right) \in \mathbb{R}^{2} \backslash\{0\} \mid e_{1}=0\right\}$. This means $V_{1}$ is a continuously decreasing function by using a Lyapunov's Theorem for Differential Inclusions [24, Prop. 14.1 p. 205]; (that does not require differentiability 
of the Lyapunov function). Since the solution of the differential equation $\dot{v}=-\gamma_{1} v^{1 / 2}-\gamma_{2} v$ is given by $v(t)=$ $\exp \left(-\gamma_{2} t\right)\left[v_{0}^{1 / 2}+\frac{\gamma_{1}}{\gamma_{2}}\left(1-\exp \left(\frac{\gamma_{2}}{2} t\right)\right)\right]$, we can conclude that the equilibrium point $\left(e_{1}, e_{2}\right)=0$ is globally finite-time stable.

\section{B. Fixed-time observer}

In this section, consider the observer (4) with the nonlinear functions $\psi_{1}$ and $\psi_{2}$ given in (5) and (6) respectively, where $\alpha, \gamma>0$ and $\beta=0$ for simplicity of the proof, which is inspired in the Lyapunov function proposed in [6].

Theorem 7: If $\gamma>0$ and $k_{1}, k_{2}, \alpha$ are selected such that (7) is satisfied, the system (4) is a global fixed-time observer for the system (3) in the sense that the estimation states $\hat{q}_{1}$ and $\hat{q}_{2}$ converge uniformly w.r.t. the initial condition and in fixed-time to the states $q_{1}$ and $q_{2}$ of system (3).

Proof: Consider a robust Lyapunov-Like function defined as

$$
V_{2}(e)=a\left|e_{1}\right|^{3}-b\left\lceil e_{1}\right\rfloor\left\lceil e_{2}\right\rfloor^{\frac{4}{3}}+c\left|e_{2}\right|^{2} .
$$

where $a, b$ and $c$ are positive.

It is necessary to show that $V_{2}$ is positive definite and $\dot{V}_{2}$ is negative definite. For this, we use the following form inequalities, derived from the Young's inequality, Lemma 3:

(i) $\quad\left|e_{1}\right|\left|e_{2}\right|^{\frac{4}{3}} \leq \frac{1}{3} \omega_{0}^{3}\left|e_{1}\right|^{3}+\frac{2}{3} \omega_{0}^{-\frac{3}{2}}\left|e_{2}\right|^{2}, \quad \forall \omega_{0}>0$,

(ii) $\quad\left|e_{1}\right|^{\frac{1}{2}}\left|e_{2}\right|^{\frac{4}{3}} \leq \frac{1}{5} \omega_{1}^{5}\left|e_{1}\right|^{\frac{5}{2}}+\frac{4}{5} \omega_{1}^{-\frac{5}{4}}\left|e_{2}\right|^{\frac{5}{3}}, \quad \forall \omega_{1}>0$

(iii) $\quad\left|e_{1}\right|^{\frac{3}{2}}\left|e_{2}\right|^{\frac{4}{3}} \leq \frac{3}{7} \omega_{2}^{\frac{7}{3}}\left|e_{1}\right|^{\frac{7}{2}}+\frac{4}{7} \omega_{2}^{-\frac{7}{4}}\left|e_{2}\right|^{\frac{7}{3}}, \quad \forall \omega_{2}>0$,

(iv) $\quad\left|e_{1}\right|\left|e_{2}\right|^{\frac{1}{3}} \leq \frac{2}{3} \omega_{3}^{\frac{3}{2}}\left|e_{1}\right|^{\frac{3}{2}}+\frac{1}{3} \omega_{4}^{-3}\left|e_{2}\right|, \quad \forall \omega_{3}>0$,

(v) $\left|e_{1}\right|^{2}\left|e_{2}\right|^{\frac{1}{3}} \leq \frac{4}{5} \omega_{4}^{\frac{5}{4}}\left|e_{1}\right|^{\frac{5}{2}}+\frac{1}{5} \omega_{4}^{-5}\left|e_{2}\right|^{\frac{5}{3}}, \quad \forall \omega_{4}>0$,

(vi) $\left|e_{1}\right|^{3}\left|e_{2}\right|^{\frac{1}{3}} \leq \frac{6}{7} \omega_{5}^{\frac{7}{6}}\left|e_{1}\right|^{\frac{7}{2}}+\frac{1}{7} \omega_{5}^{-7}\left|e_{2}\right|^{\frac{7}{3}}, \quad \forall \omega_{5}>0$,

(vii) $\quad\left|e_{1}\right|\left|e_{2}\right| \leq \frac{2}{5} \omega_{6}^{\frac{5}{2}}\left|e_{1}\right|^{\frac{5}{2}}+\frac{3}{5} \omega_{6}^{-\frac{5}{3}}\left|e_{2}\right|^{\frac{5}{3}}, \quad \forall \omega_{6}>0$.

From inequality $(i)$, the function $V_{2}$ satisfies

$$
\tilde{a}_{1}\left|e_{1}\right|^{3}+\tilde{a}_{2}\left|e_{2}\right|^{2} \leq V_{2}(e) \leq \tilde{b}_{1}\left|e_{1}\right|^{3}+\tilde{b}_{2}\left|e_{1}\right|^{2}
$$

where $\quad \tilde{a}_{1}=\left(a-\frac{b \omega_{0}^{3}}{3}\right), \quad \tilde{a}_{2}=\left(c-\frac{2 b \omega_{0}^{-\frac{3}{2}}}{3}\right)$, $\tilde{b}_{1}=\left(a+\frac{b \omega_{0}^{3}}{3}\right)$, and $\tilde{b}_{2}=\left(c+\frac{2 b \omega_{0}^{-\frac{3}{2}}}{3}\right)$. Therefore, $V_{2}>0$ if $a>\frac{b}{3} \omega_{0}^{3}$ and $c>\frac{2}{3} b \omega_{0}^{-\frac{3}{2}}$.

The derivative of $V_{2}$ along the trajectories of (11) is given by

$$
\begin{aligned}
\dot{V}_{2}(e)= & \frac{1}{\sqrt{m\left(q_{1}\right)}}\left\{-3 a k_{1} \alpha^{2}\left|e_{1}\right|^{5 / 2}-3 a \alpha k_{1} \gamma\left|e_{1}\right|^{7 / 2}\right. \\
& -b\left|e_{2}\right|^{\frac{7}{3}}+3 a \alpha\left\lceil e_{1}\right\rfloor^{2} e_{2}+b k_{1} \alpha\left\lceil e_{1}\right\rfloor^{\frac{1}{2}}\left\lceil e_{2}\right\rfloor^{\frac{4}{3}} \\
& +b k_{1} \gamma\left\lceil e_{1}\right\rfloor^{\frac{3}{2}}\left\lceil e_{2}\right\rfloor^{\frac{4}{3}}+\frac{2}{3} b k_{2} \alpha^{2}\left|e_{1}\right|\left|e_{2}\right|^{\frac{1}{3}} \\
& +\frac{8}{3} b k_{2} \alpha \gamma\left|e_{1}\right|^{2}\left|e_{2}\right|^{\frac{1}{3}}+2 b k_{2} \gamma^{2}\left|e_{1}\right|^{3}\left|e_{2}\right|^{\frac{1}{3}} \\
& -c k_{2} \alpha^{2}\left\lceil e_{1}\right\rfloor^{0} e_{2}-4 c k_{2} \alpha \gamma e_{1} e_{2} \\
& \left.-3 c k_{2} \gamma^{2}\left\lceil e_{1}\right\rfloor^{2} e_{2}-\frac{4}{3} b \delta\left\lceil e_{1}\right\rfloor\left\lceil e_{2}\right\rfloor^{\frac{1}{3}}+2 c \delta e_{2}\right\}
\end{aligned}
$$

which can be bounded using absolute value as follows, where $a$ is selected as $a=\frac{c k_{2} \gamma^{2}}{\alpha}$ for canceling the term $\left\lceil e_{1}\right\rfloor^{2} e_{2}$ and $c$ is selected sufficiently large:

$$
\begin{aligned}
\dot{V}_{2}(e) \leq & \frac{1}{\sqrt{m\left(q_{1}\right)}}\left\{-3 c k_{1} k_{2} \alpha \gamma^{2}\left|e_{1}\right|^{\frac{5}{2}}-3 c k_{1} k_{2} \gamma^{3}\left|e_{1}\right|^{\frac{7}{2}}\right. \\
& -b\left|e_{2}\right|^{\frac{7}{3}}+b k_{1} \alpha\left|e_{1}\right|^{\frac{1}{2}}\left|e_{2}\right|^{\frac{4}{3}}+b k_{1} \gamma\left|e_{1}\right|^{\frac{3}{2}}\left|e_{2}\right|^{\frac{4}{3}} \\
& +\left(\frac{2}{3} b k_{2} \alpha^{2}+\frac{4}{3} b L_{\delta}\right)\left|e_{1}\right|\left|e_{2}\right|^{\frac{1}{3}}+\frac{8}{3} b k_{2} \alpha \gamma\left|e_{1}\right|^{2}\left|e_{2}\right|^{\frac{1}{3}} \\
& +2 b k_{2} \gamma^{2}\left|e_{1}\right|^{3}\left|e_{2}\right|^{\frac{1}{3}}+\left(c k_{2} \alpha^{2}+2 c L_{\delta}\right)\left|e_{2}\right| \\
& \left.+4 c k_{2} \alpha \gamma\left|e_{1}\right|\left|e_{2}\right|\right\} .
\end{aligned}
$$

From inequalities $(i i)-(v i i)$, the previous inequality leads to the next inequality

$$
\begin{aligned}
\dot{V}_{2}(e) \leq \frac{1}{\sqrt{m\left(q_{1}\right)}}\left\{-\vartheta_{1}\left|e_{1}\right|^{\frac{7}{2}}-\vartheta_{2}\left|e_{2}\right|^{\frac{7}{3}}\right. & +\vartheta_{3}\left(\left|e_{1}\right|^{\frac{5}{2}}+\left|e_{2}\right|^{\frac{5}{3}}\right) \\
& \left.+\vartheta_{4}\left(\left|e_{1}\right|^{\frac{3}{2}}+\left|e_{2}\right|\right)\right\}
\end{aligned}
$$

where

$$
\begin{aligned}
\vartheta_{1} & =3 c k_{1} k_{2} \gamma^{3}-\frac{3}{7} b k_{1} \gamma \omega_{2}^{\frac{7}{3}}-\frac{12}{7} b k_{2} \gamma^{2} \omega_{5}^{\frac{7}{6}}, \\
\vartheta_{2} & =b-\frac{4}{7} b k_{1} \gamma \omega_{2}^{-\frac{7}{4}}-\frac{2}{7} b k_{2} \gamma^{2} \omega_{5}^{-7}, \\
\vartheta_{3} & =\max \left\{\varsigma_{3}, \tilde{\varsigma_{3}}\right\} \\
\vartheta_{4} & =\max \left\{\varsigma_{4}, \tilde{\varsigma_{4}}\right\} \\
\varsigma_{3} & =-c k_{2} \alpha \gamma\left(3 k_{1} \gamma-\frac{4}{5} \omega_{6}^{\frac{5}{2}}\right)+\frac{1}{5} b k_{1} \alpha \omega_{1}^{5}+\frac{16}{15} b k_{2} \alpha \gamma \omega_{4}^{\frac{5}{4}}, \\
\tilde{\varsigma_{3}} & =\frac{4}{5} b k_{1} \alpha \omega_{1}^{-\frac{5}{4}}+\frac{4}{15} b k_{2} \alpha \gamma \omega_{4}^{-5}+\frac{6}{5} c k_{2} \alpha \gamma \omega_{6}^{-\frac{5}{3}}, \\
\varsigma_{4} & =\frac{4}{9}\left(b k_{2} \alpha^{2}+2 b L_{\delta}\right) \omega_{3}^{\frac{3}{2}}, \\
\tilde{\varsigma_{4}} & =c\left(k_{2} \alpha^{2}+2 L_{\delta}\right)+\frac{2}{9} b\left(k_{2} \alpha^{2}+2 L_{\delta}\right) \omega_{4}^{-3} .
\end{aligned}
$$

We recall the fundamental inequality proposed in [25, Thm. 16, Section 2.9]

$$
\left(\tilde{\sigma}\left|x_{1}\right|^{s_{1}}+(1-\tilde{\sigma})\left|x_{2}\right|^{s_{1}}\right)^{\frac{1}{s_{1}}} \leq\left(\tilde{\sigma}\left|x_{1}\right|^{s_{2}}+(1-\tilde{\sigma})\left|x_{2}\right|^{s_{2}}\right)^{\frac{1}{s_{2}}}
$$


$0<\tilde{\sigma}<1, \forall x \in \mathbb{R}^{2}, 0<s_{1} \leq s_{2}$. This inequality implies

$$
\begin{gathered}
\frac{1}{2}\left(\left|e_{1}\right|^{\frac{5}{2}}+\left|e_{2}\right|^{\frac{5}{3}}\right) \leq \frac{1}{2^{\frac{5}{7}}}\left(\left|e_{1}\right|^{\frac{7}{2}}+\left|e_{2}\right|^{\frac{7}{3}}\right)^{\frac{5}{7}} \\
\frac{1}{2}\left(\left|e_{1}\right|^{\frac{3}{2}}+\left|e_{2}\right|\right) \leq \frac{1}{2^{\frac{3}{7}}}\left(\left|e_{1}\right|^{\frac{7}{2}}+\left|e_{2}\right|^{\frac{7}{3}}\right)^{\frac{3}{7}}
\end{gathered}
$$

which imply that $\dot{V}_{2}$ satisfies

$$
\begin{aligned}
\dot{V}_{2}(e) & \leq-\vartheta_{5}\|e\|_{h}^{\frac{7}{2}}+\vartheta_{6}\|e\|_{h}^{\frac{5}{2}}+\vartheta_{7}\|e\|_{h}^{\frac{3}{2}} \\
& =-\sigma \vartheta_{5}\|e\|_{h}^{\frac{7}{2}}-\|e\|_{h}^{\frac{3}{2}}\left[(1-\sigma) \vartheta_{5}\|e\|_{h}^{2}-\vartheta_{6}\|e\|_{h}-\vartheta_{7}\right]
\end{aligned}
$$

where $0<\sigma<1$, $\|e\|_{h}=\left(\left|e_{1}\right|^{\frac{7}{2}}+\left|e_{2}\right|^{\frac{7}{3}}\right)^{\frac{2}{7}}$ and $\vartheta_{5}=$ $\min \left\{\vartheta_{1}, \vartheta_{2}\right\}, \vartheta_{6}=2^{\frac{2}{7}} \vartheta_{3}, \vartheta_{7}=2^{\frac{4}{7}} \vartheta_{4}$. We can ensure that:

$$
\dot{V}_{2}(e) \leq-\sigma \vartheta_{5}\|e\|_{h}^{\frac{7}{2}} \leq-\vartheta_{8} V_{2}^{\frac{7}{6}}(e)
$$

for all $e \in \mathscr{C}=\left\{e \in \mathbb{R}^{2} \mid\|e\|_{h} \geq \frac{\vartheta_{6}+\sqrt{\vartheta_{6}^{2}+4(1-\sigma) \vartheta_{7}}}{2(1-\sigma)}\right\}$ and $\vartheta_{8}=2^{-\frac{1}{6}} \sigma \vartheta_{5} \tilde{c}^{-\frac{7}{6}}$ with $\tilde{c}=\max \left\{\tilde{b}_{1}, \tilde{b}_{2}\right\}$.

Since the solution of the differential equation $\dot{v}=-\gamma v^{\frac{7}{6}}$ is given by $v(t)=\left(v_{0}^{-\frac{1}{6}}+\frac{\gamma}{6} t\right)^{-6}$, then, from (21) and comparison principle, $V_{2}(e(t)) \leq\left(V_{2}(e(0))^{-\frac{1}{6}}+\frac{\vartheta_{8}}{6} t\right)^{-6}$ which implies $V_{2}(e(t)) \leq V_{2}(e(0))$. Put $V_{2}(e(t))=\tilde{k} \geq \frac{\vartheta_{6}+\sqrt{\vartheta_{6}^{2}+4(1-\sigma) \vartheta_{7}}}{2(1-\sigma)}$, the time $T_{\tilde{k}}$ to go from $V_{2}(e(0))$ to $\tilde{k}$ is bounded by $\frac{6}{\vartheta_{8}}\left(\tilde{k}^{-\frac{1}{6}}-V_{2}(e(0))^{-\frac{1}{6}}\right)$, which can be bounded independently of the initial condition $V_{2}\left(e_{2}(0)\right)$, i.e. $T_{\tilde{k}} \leq \frac{6}{\vartheta_{8}} \tilde{k}^{-\frac{1}{6}}$. This implies that the trajectories of the system (11) reach the compact set $\tilde{\mathscr{C}}=\left\{e \in \mathbb{R}^{2} \mid\|e\|_{h} \geq \tilde{k}\right\}$ in a time bounded by $\frac{6}{\vartheta_{8}} \tilde{k}^{-\frac{1}{6}}$ independently of the initial condition. The time to reach $e=0$ with initial conditions in $\tilde{\mathscr{C}}$ can be bounded by the compactness of $\tilde{\mathscr{C}}$. Thus, we can conclude that the equilibrium point $\left(e_{1}, e_{2}\right)=0$ is globally fixed-time stable.

\section{Simulation EXAMPLE}

Consider an inverted pendulum on a cart which is adapted from [26]

$$
\begin{aligned}
\left(1-\frac{3 \varepsilon}{4} \cos ^{2}(q)\right) \ddot{q} & +\frac{3 \varepsilon}{8} \dot{q}^{2} \sin (2 q) \\
& -\sin (q)-u \cos (q)+\delta=0 .
\end{aligned}
$$

where $m$ and $M$ are the pendulum mass and cart mass respectively, and $\varepsilon=\frac{m}{m+M}$ is the relative mass, $u$ is the control law that is the horizontal driving force and $\delta$ represents the disturbance. Assumption (A-1) is satisfied with $\varsigma_{m}=1-\frac{3 \varepsilon}{4}$ and $\varsigma_{M}=1,(\mathrm{~A}-2)$ can be straightforwardly verified from trigonometric properties.

For the numerical results reported bellow, the following parameters were chosen solving the matrix inequality (7) with disturbance bound $L_{\delta}=0.5: P=\left[\begin{array}{cc}0.78 & -0.4 \\ -0.4 & 0.37\end{array}\right], A=$ $\left[\begin{array}{rr}-1.956 & 1 \\ -1.87 & 0\end{array}\right], \rho=1$, and $\alpha=1.1$. The pendulum mass and cart

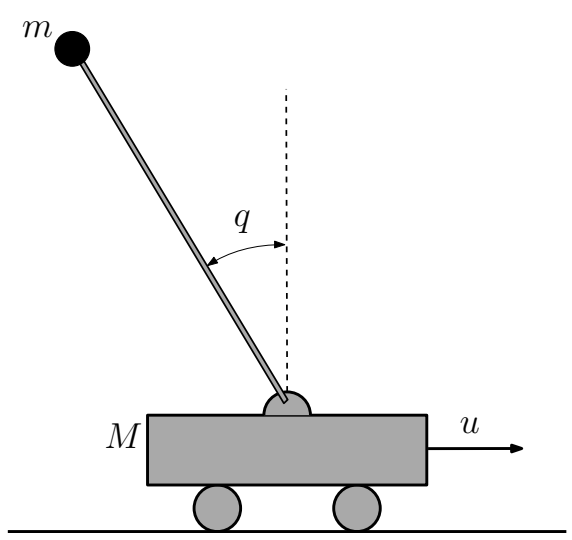

Fig. 1. Inverted pendulum on a cart.

mass are chosen as $m=0.2, M=1$. The proposed observer is given by

$$
\left\{\begin{array}{r}
\dot{\hat{q}}_{1}=\frac{1}{\sqrt{m\left(q_{1}\right)}}\left(\sqrt{m\left(\hat{q}_{1}\right)} \hat{q}_{2}+1.95 \cdot \psi_{1}\left(e_{1}\right)\right) \\
\dot{\hat{q}}_{2}=\frac{1}{\sqrt{m\left(\hat{q}_{1}\right) m\left(q_{1}\right)}}\left(u-g\left(q_{1}\right)+1.87 \psi_{2}\left(q_{1}-\hat{q}_{1}\right)\right) \\
-\frac{1}{m\left(\hat{q}_{1}\right)} c\left(\hat{q}_{1}\right) \hat{q}_{2}^{2}
\end{array}\right.
$$

where $m\left(q_{1}\right)=1-\frac{3 \varepsilon}{4} \cos ^{2}\left(q_{1}\right), m\left(\hat{q}_{1}\right)=1-\frac{3 \varepsilon}{4} \cos ^{2}\left(\hat{q}_{1}\right)$, $c\left(\hat{q}_{1}\right)=\frac{3 \varepsilon}{8} \sin \left(2 q_{1}\right), \psi_{1}\left(e_{1}\right)=1.1\left\lceil e_{1}\right\rfloor^{\frac{1}{2}}+\left\lceil e_{1}\right\rfloor$ and $\psi_{2}\left(e_{1}\right)=$ $\frac{(1.1)^{2}}{2}\left\lceil e_{1}\right\rfloor^{0}+\frac{3(1.1)}{2}\left\lceil e_{1}\right\rfloor^{\frac{1}{2}}+\left\lceil e_{1}\right\rfloor$.

Figure 2. presents the simulation results for finite-time observer for initial conditions $q(0)=0.5$ and $\hat{q}(0)=5$. Note that the considered mechanical system is unstable, but the convergence of the observed state to the real state of the mechanical system is guaranteed in a finite-time (less than 2 seconds).

\section{CONCLUSION}

In this paper, we propose a global sliding mode observer for a class of mechanical systems with Coriolis and centrifugal forces and disturbances. The proposed observer does not require of a prior transformation and provides finite-time and fixed-time convergence to the position and velocity of the mechanical system. BIBS stability of mechanical system is not required for the observer design, and its gains can be obtained from a feasible matrix inequality. Future work will focus on extending the approach to other types of specifications and to other types scenarios such as time-delay in the framework of Multiplicity-Induced-Dominancy (MID) properties [27].

\section{REFERENCES}

[1] M.W. Spong, S. Hutchinson, and M. Vidyasagar. Robot Modeling and Control. Wiley select coursepack. Wiley, 2005.

[2] R. Ortega, J.A. Loría Perez, P.J. Nicklasson, and H. H. Sira-Ramirez. Passivity-based Control of Euler-Lagrange Systems: mechanical, electrical and Electromechanical applications. Springer, London, 1998.

[3] A. Levant. Robust exact differentiation via sliding mode technique. Automatica, 34(3):379-384, 1998.

[4] J. Davila, L. Fridman, and A. Levant. Second-order sliding-mode observer for mechanical systems. IEEE Transactions on Automatic Control, 50(11):1785-1789, 2005. 

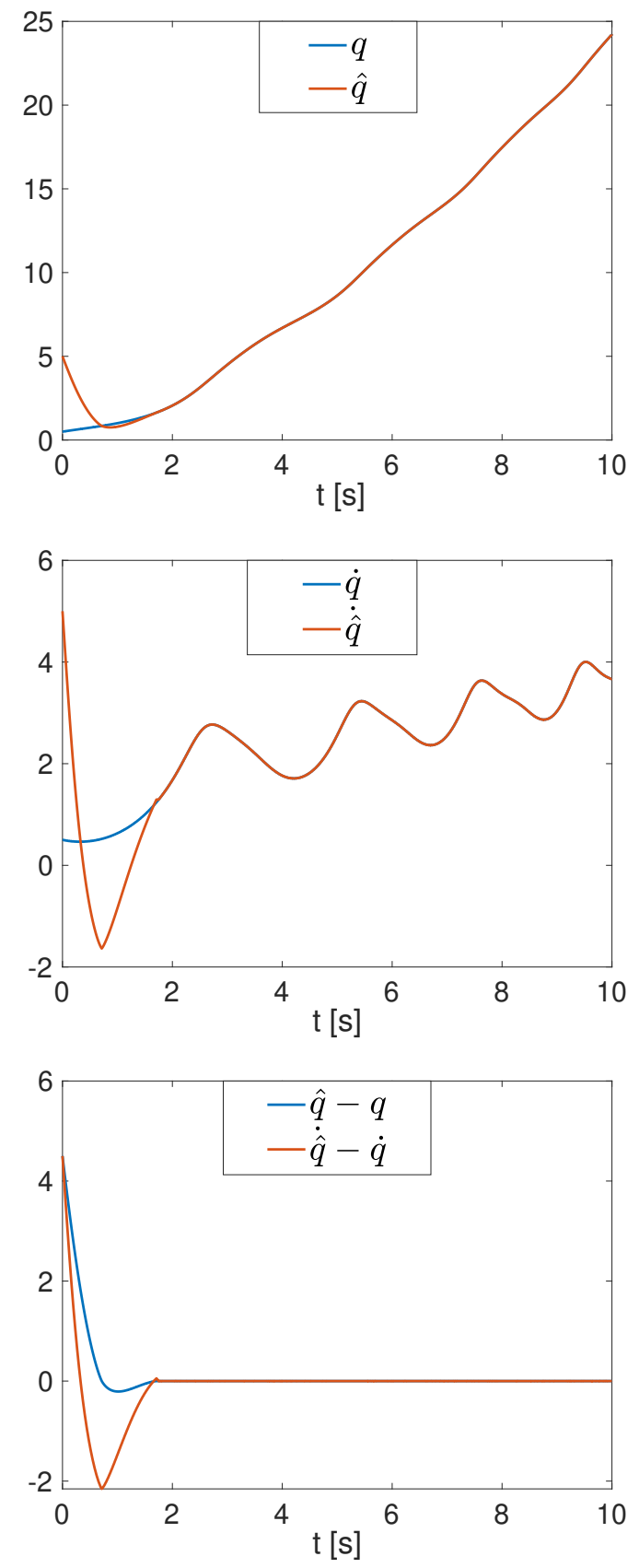

Fig. 2. Position, Velocity and Errors of system (22)

[5] L. Fridman, Y. Shtessel, C. Edwards, and Xing-Gang X.-G. Yan. Higher-order sliding-mode observer for state estimation and input reconstruction in nonlinear systems. International Journal of Robust and Nonlinear Control, 18(4-5):399-412, 2008.

[6] E. Cruz-Zavala, J. A. Moreno, and L. M. Fridman. Uniform robust exact differentiator. IEEE Transactions on Automatic Control, 56(11):2727-2733, 2011.

[7] T. R. Oliveira, A. Estrada, and L. M. Fridman. Global and exact hosm differentiator with dynamic gains for output-feedback sliding mode control. Automatica, 81:156-163, 2017.

[8] W.A. Apaza-Perez, J.A. Moreno, and L.M. Fridman. Dissipative approach to sliding mode observers design for uncertain mechanical systems. Automatica, 87:330-336, 2018.

[9] M.L.J. Hautus. Strong detectability and observers. Linear Algebra and its Applications, 50:353-368, 1983.

[10] E. Rocha-Cózat and J. A. Moreno. Dissipativity and design of unknown input observers for nonlinear systems. IFAC Proceedings Volumes, 37(13):471-476, 2004. 6th IFAC Symposium on Nonlinear Control Systems 2004 (NOLCOS 2004), Stuttgart, Germany, 1-3 September, 2004.

[11] J.A. Moreno. Approximate Observer Error Linearization by Dissipativity Methods, pages 35-51. Springer Berlin Heidelberg, Berlin, Heidelberg, 2005.

[12] Edmundo Rocha-Cózatl and Jaime A. Moreno. Dissipative design of unknown input observers for systems with sector nonlinearities. International Journal of Robust and Nonlinear Control, 21(14):16231644, 2011.

[13] G. Besançon. Global output feedback tracking control for a class of lagrangian systems. Automatica, 36(12):1915-1921, 2000.

[14] M. Mabrouk, F. Mazenc, and J.-C. Vivalda. On global observers for some mechanical systems. IFAC Proceedings Volumes, 37(21):675680, 2004. 2nd IFAC Symposium on System Structure and Control, Oaxaca, Mexico, December 8-10, 2004.

[15] A. Astolfi, R. Ortega, and A. Venkatraman. A globally exponentially convergent immersion and invariance speed observer for mechanical systems with non-holonomic constraints. Automatica, 46(1):182-189, 2010.

[16] Ø.N. Stamnes, O.M. Aamo, and G.-. Kaasa. A constructive speed observer design for general euler-lagrange systems. Automatica, 47(10):2233-2238, 2011.

[17] C. Edwards, P. Menon, and Y. Shtessel. Super-twisting observation for a class of lagrangian systems. In 2017 IEEE 56th Annual Conference on Decision and Control (CDC), pages 6045-6050, 2017.

[18] W. A. Apaza-Perez, J. A. Moreno, and L. Fridman. Global sliding mode observers for some uncertain mechanical systems. IEEE Transactions on Automatic Control, 65(3):1348-1355, 2020.

[19] Sanjay P Bhat and Dennis S Bernstein. Finite-time stability of continuous autonomous systems. SIAM Journal on Control and Optimization, 38(3):751-766, 2000.

[20] Andrey Polyakov. Nonlinear feedback design for fixed-time stabilization of linear control systems. IEEE Transactions on Automatic Control, 57(8):2106-2110, 2011.

[21] G.H. Hardy, J.E. Littlewood, and G. Polya. Inequalities. Cambridge Univ. Press, London,U.K., 1951

[22] A. F. Filippov. Differential Equations with Discontinuous Righthand Sides: Control Systems (Mathematics and its Applications). Springer, Netherlands, 1988.

[23] J.A. Moreno. A linear framework for the robust stability analysis of a generalized super-twisting algorithm. In 2009 6th International Conference on Electrical Engineering, Computing Science and Automatic Control (CCE), pages 1-6, 2009.

[24] K. Deimling. Multivalued Differential Equations. Walter de Gruyter, Berlin, 1992.

[25] L. Rosier and A. Bacciotti. Liapunov functions and stability in control theory. Second edition. Springer Verlag, 2005.

[26] J. Sieber and B. Krauskopf. Extending the permissible control loop latency for the controlled inverted pendulum. Dynamical Systems, 20(2):189-199, 2005

[27] A. Benarab, I. Boussaada, K. Trabelsi, G. Mazanti, and C. Bonnet The mid property for a second-order neutral time-delay differential equation. In 2020 24th International Conference on System Theory, Control and Computing (ICSTCC), pages 7-12, 2020. 